\title{
First-Principles Study on the Electronic Structure and Elastic Properties of YCu, DyCu and YAg
}

\author{
Y. J. Shi ${ }^{1}$, Y. L. Du ${ }^{1, *}$, G. Chen ${ }^{1}$ and G. L. Chen ${ }^{1,2}$ \\ ${ }^{1}$ Department of Materials Science and Engineering, Nanjing University of Science and Technology, \\ Nanjing 210094, P.R. China \\ ${ }^{2}$ State Key Laboratory for Advanced Metals and Materials, University of Science and Technology Beijing, \\ Beijing 100083, P.R. China
}

The electronic structure and elastic properties of $\mathrm{YCu}, \mathrm{YAg}$, and $\mathrm{DyCu}$ were studied by full-potential linearized augmented plane wave method (FPLAPW) on the basis of the density functional theory (DFT). The generalized gradient approximation (GGA) is applied for YCu, $\mathrm{YAg}$, and the $\mathrm{LDA}+\mathrm{U}$ is applied for DyCu. The density of states at the Fermi energy, $N\left(E_{\mathrm{F}}\right)$, are 1.08, 1.09, and 1.04 states/(eV unit cell) for $\mathrm{YCu}, \mathrm{YAg}$ and $\mathrm{DyCu}$, respectively. The elastic constants were calculated. The values for Pugh's criterion are 2.30, 2.05 and $3.00 \mathrm{for} \mathrm{YCu}, \mathrm{DyCu}$ and YAg, respectively. All of them are larger than 1.75, indicating the ductile manner of these materials. The calculated value of the anisotropy is close to 1, indicating highly isotropic behavior. [doi:10.2320/matertrans.MB200816]

(Received May 1, 2008; Accepted August 25, 2008; Published October 8, 2008)

Keywords: first-principles, electronic structure, elastic properties

\section{Introduction}

The B2 (CsCl-type structure) intermetallic compounds have been extensively studied for several decades due to their high oxidation resistance, high stiffness and high strength for elevated temperature engineering applications. ${ }^{1-3)}$ Most of the B2 polycrystalline intermetallic compounds usually possess little or no tensile ductility at room temperature if they are fully ordered and exactly stoichiometric. ${ }^{1-3)}$ However, a new family of fully ordered, stoichiometric binary rare-earth intermetallic compounds denoted as $R M$ (where $R$ is a rare-earth element, and $M$ is a main group or transition metal element) has recently been found to be ductile at room temperature. ${ }^{4)}$ For example, the tensile testing of polycrystalline $\mathrm{YCu}$ specimens produces elongations as high as $11 \%$ before failure; $\mathrm{DyCu}$ has an intermediate ductility, $16 \%$ elongation before failure; while $\mathrm{YAg}$ is the most ductile, producing $20 \%$ elongations before failure. ${ }^{5)}$ Besides $\mathrm{YCu}$, $\mathrm{DyCu}$ and $\mathrm{YAg}$, nine other $R M$ intermetallics are also ductile. ${ }^{4)}$ These $R M$ intermetallics with high ductility are promising high-temperature structural materials due to their high-temperature strength and corrosion resistance. Furthermore, the studies on the mechanism of the high ductility in $R M$ intermetallics may offer insights into alloy modifications that could improve the ductility of other intermetallic compounds. ${ }^{5-7)}$

So far, many experimental works have been done to reveal the origin of the high ductility in $R M$ intermetallics. However, the mechanism has not been well understood. In addition to the experimental work, first-principles calculations based on density functional theory (DFT) have been used to reveal the electronic structure, structural and physical properties of $R M$. For example, the bulk and defect properties of $\mathrm{YAg}$ and $\mathrm{YCu}$ have been calculated to explain the ductility. ${ }^{8)}$ And the phase stability and pressure-induced phase transition in $\mathrm{YCu}$ were also studied.9) As is known, $\mathrm{YCu}, \mathrm{DyCu}$ and $\mathrm{YAg}$ have the same crystal structure, but

*Corresponding author, E-mail: yldu_njust@126.com they show different room-temperature ductility. The comparative analysis of electronic structure may be beneficial to understand the different ductility in $R M$ intermetallics. However, as a typical member of ductile $R M$, the electronic structure of DyCu has not been studied due to the partially filled $4 f$ level in Dy atom, which usually results in absurd calculation results. Fortunately, the recently developed LDA $+\mathrm{U}$ method takes into account orbital dependence of the Coulomb and exchange interactions which is absent in the LDA or GGA, and is known to give a correct description of the systems where $f$-electrons are well localized. ${ }^{10,11)}$ In this paper, we present first-principles calculations of $\mathrm{YCu}, \mathrm{DyCu}$ and YAg. The comparative analysis of their electronic structures and elastic properties were performed. The ductile behavior of these intermetallics is predicted on the basis of the calculated results. The aim of our work is to extend the understanding of the anomalous ductility in $R M$ intermetallics.

\section{Computational Method}

The presented calculations were performed by using the full-potential linearized augmented plane wave method (FP_LAPW) as implemented in the latest WIEN2k code. ${ }^{12)}$ The structures of $\mathrm{YCu}, \mathrm{DyCu}$ and $\mathrm{YAg}$ are all primitive cubic crystal lattice, with B2 (CsCl-type) structure, belonging to the space group $P m \overline{3} m .^{4,13)}$ Figure 1 illustrates the crystal structure of $R M$. It is clear that the $M$ atoms are positioned at the center of the cubic cell $(0.5,0.5,0.5)$ and $R$ atoms occupy $(0,0,0)$ positions at the eight corners of the cubic cell. In our calculations, the exchange and correlation effects are treated using the generalized gradient approximation (GGA) for $\mathrm{YCu}$ and $\mathrm{YAg}$, while the LDA+U method is used for DyCu. It is known that Dy atom with localized $4 f$ electrons is belong to strongly correlated systems. The generalized gradient approximation (GGA) can not be applied for strongly correlated systems, however, the recently developed LDA $+\mathrm{U}$ method takes into account orbital dependence of the Coulomb and exchange interactions which is absent in 


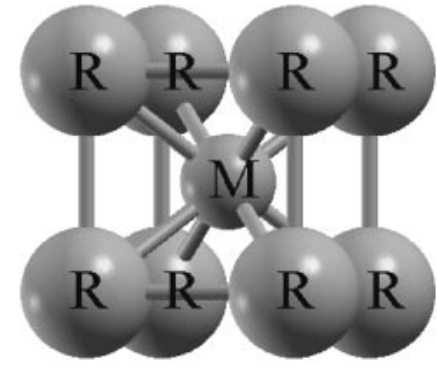

Fig. 1 Crystal structure of $R M$ intermetallics.

the GGA and is known to give a correct description of the strongly correlated systems. ${ }^{10)}$ The radii of spheres for Y, Dy, $\mathrm{Cu}$ and $\mathrm{Ag}$ have been chosen as 2.5, 2.7, 2.2 and 2.5 atomic units, respectively. The energy that separates the valence states from the core states were all chosen to be -6.0 Ryd $(-81.6 \mathrm{eV}) .1000 \mathrm{k}$-points are used in the whole Brillouin zone. During the self-consistency cycle, the total energy is converged to within $10 \mu \mathrm{Ry} / \mathrm{f}$.u. In the $\mathrm{LDA}+\mathrm{U}$ method, $\mathrm{U}$ is set as $0.52 \mathrm{Ry}$ for $\mathrm{DyCu}$. There are three independent elastic constants for cubic crystal, $C_{11}, C_{12}$ and $C_{44}$. In the present work, the elastic constants were calculated by the strainenergy method. An appropriate set of strains was applied to the undeformed unit cell lattice with the relaxed structure. Then, the elastic constants were determined from the resulting change in total energy on the deformation. ${ }^{14,15)}$

\section{Results and Discussion}

As a first step, the total energies of $\mathrm{YCu}, \mathrm{DyCu}$ and $\mathrm{YAg}$ with different volumes are calculated respectively in order to determine the equilibrium structural parameters. This is performed by applying hydrostatic pressure by varying volume with the constant ratio of $a: b: c=1: 1: 1$. The point of crystal energy minimum is corresponding to the equilibrium. The volume variations applied were $-10,-5,0$, +5 and $+10 \%$ of the experimental volume. The energy vs. volume curves of $\mathrm{YCu}, \mathrm{DyCu}$ and $\mathrm{YAg}$ are shown in Fig. 2. The equilibrium lattice constant can be obtained by fitting the total energy vs. volume data to Murnaghan equation. ${ }^{16)}$ The results are listed in Table 1. For comparison, the corresponding experimental values are also presented. The calculated equilibrium lattice constant of $\mathrm{YCu}, \mathrm{DyCu}$ and $\mathrm{YAg}$ are $3.473,3.459$ and $3.632 \AA$, respectively. For calculated equilibrium volumes, the values are 41.882, 41.403 and $47.920 \AA^{3}$ for $\mathrm{YCu}$, DyCu and YAg respectively. As shown in the table, the calculated lattice constants and equilibrium volumes are in good agreement with those experimental values. Thus, we can conclude that the computation parameter and conditions selected in this calculated work should be suitable.

The total and partial densities of states (DOS) of $\mathrm{YCu}$, YAg and $\mathrm{DyCu}$ are plotted in Figs. 3(a), (b) and (c), respectively. It can be seen that the DOSs at the Fermi level are all higher than zero, 1.08 states/(eV unit cell) for $\mathrm{YCu}$, 1.09 states/(eV unit cell) for YAg, and 1.04 states/(eV unit cell) for $\mathrm{DyCu}$, respectively. Hence, they have obvious metallic character. ${ }^{17)}$ As shown in the figure, for $\mathrm{YCu}$ and $\mathrm{DyCu}$, the large occupied peak is located at about $-3.0 \mathrm{eV}$

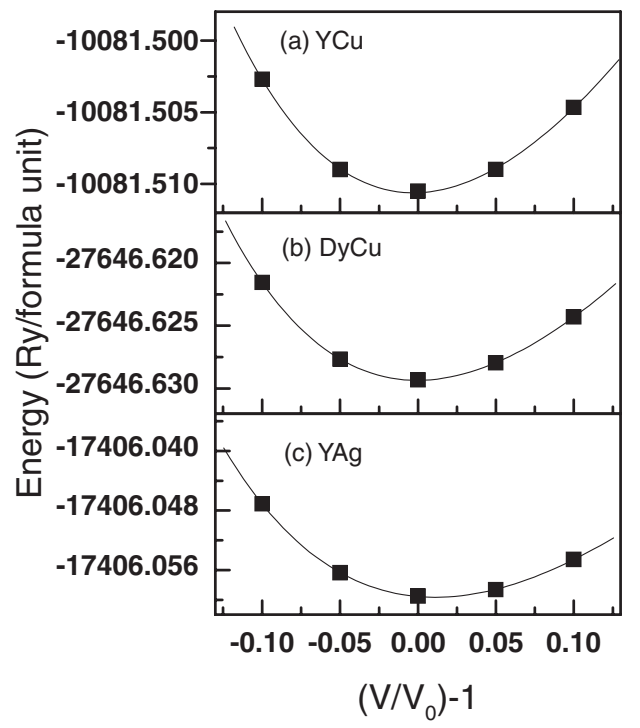

Fig. 2 Calculated total energy vs. relative volume/f.u. of (a) $\mathrm{YCu}$, (b) DyCu and (c) YAg. $V_{0}$ is the corresponding experimental volume.

Table 1 The experimental and calculated structural parameters for $\mathrm{YCu}$, DyCu and YAg, respectively.

\begin{tabular}{|c|c|c|c|}
\hline & $R M$ & $a(\AA)$ & Volume $\left(\AA^{3}\right)$ \\
\hline \multirow{2}{*}{$\mathrm{YCu}$} & Experimental $^{13)}$ & 3.476 & 41.999 \\
\hline & Present calculation & 3.473 & 41.882 \\
\hline \multirow{2}{*}{$\mathrm{DyCu}$} & Experimental $^{4)}$ & 3.460 & 41.422 \\
\hline & Present calculation & 3.459 & 41.403 \\
\hline \multirow{2}{*}{ YAg } & Experimental $^{13)}$ & 3.619 & 47.399 \\
\hline & Present calculation & 3.632 & 47.920 \\
\hline
\end{tabular}

below the Fermi level, which is dominated by the $\mathrm{Cu} d$-state according to the results of partial DOSs. But for YAg, the large occupied peak is to shift to the energy $-5.0 \mathrm{eV}$, which is dominated by the $\mathrm{Ag} d$-state. The states above the Fermi level are mainly due to $\mathrm{Y} d$-state in $\mathrm{YCu}$ and $\mathrm{YAg}$, and Dy $d$-state in DyCu, respectively. In binary alloys, it is important to analyze the bonding character near the Fermi level. As shown in Fig. 3, the Fermi energy of $\mathrm{YCu}, \mathrm{DyCu}$ and $\mathrm{YAg}$ lies near a local minimum, which means that the bonding is relatively insensitive to local distortions. ${ }^{18)}$ In contrast, the Fermi energy of NiAl, a typical intermetallic with brittle behavior, is located at the peak in the DOS. Thus, the difference in ductility between RM ductile intermetallics and the other brittle intermetallics may be partially explained by their difference in Fermi level.

Knowledge of the elastic properties of materials promotes the understanding of the fundamental aspects of mechanical deformation and structural properties of crystals. Above all, we can predict the mechanical properties by the elastic constants, particularly, the ductility/brittleness of the materials. The calculated elastic constants of $\mathrm{YCu}, \mathrm{DyCu}$ and YAg have been listed in Table 2. For a comparison, the corresponding experimental values are also presented. From Table 2, it is analyzed that the calculated elastic constants of $\mathrm{YCu}$ and $\mathrm{YAg}$ are very close to the experimental value. On the basis of the fact, though it is lack of reports for $\mathrm{DyCu}$, we should think that the calculated values of elastic constants for $\mathrm{DyCu}$ are credible. 
Table 2 The calculated and experimental elastic constants, bulk modulus $K$, anisotropy, and Poisson's ratio for YCu, YAg and DyCu.

\begin{tabular}{|c|c|c|c|c|c|}
\hline & \multicolumn{2}{|c|}{$\mathrm{YCu}$} & \multicolumn{2}{|c|}{ YAg } & \multirow{2}{*}{$\begin{array}{c}\mathrm{DyCu} \\
\text { Calculated }\end{array}$} \\
\hline & Calculated & Experimental $^{8)}$ & Calculated & Experimental $^{8)}$ & \\
\hline$C_{11}(\mathrm{GPa})$ & 110.7 & 113.4 & 107.3 & 102.4 & 106.1 \\
\hline$C_{12}(\mathrm{GPa})$ & 50.7 & 48.4 & 58.4 & 54.0 & 42.3 \\
\hline$K(\mathrm{GPa})$ & 70.7 & 70.1 & 74.7 & 70.5 & 63.6 \\
\hline Anisotropy & 1.18 & 0.99 & 1.55 & 1.54 & 1.45 \\
\hline$v$ & 0.31 & 0.30 & 0.35 & 0.35 & 0.29 \\
\hline
\end{tabular}
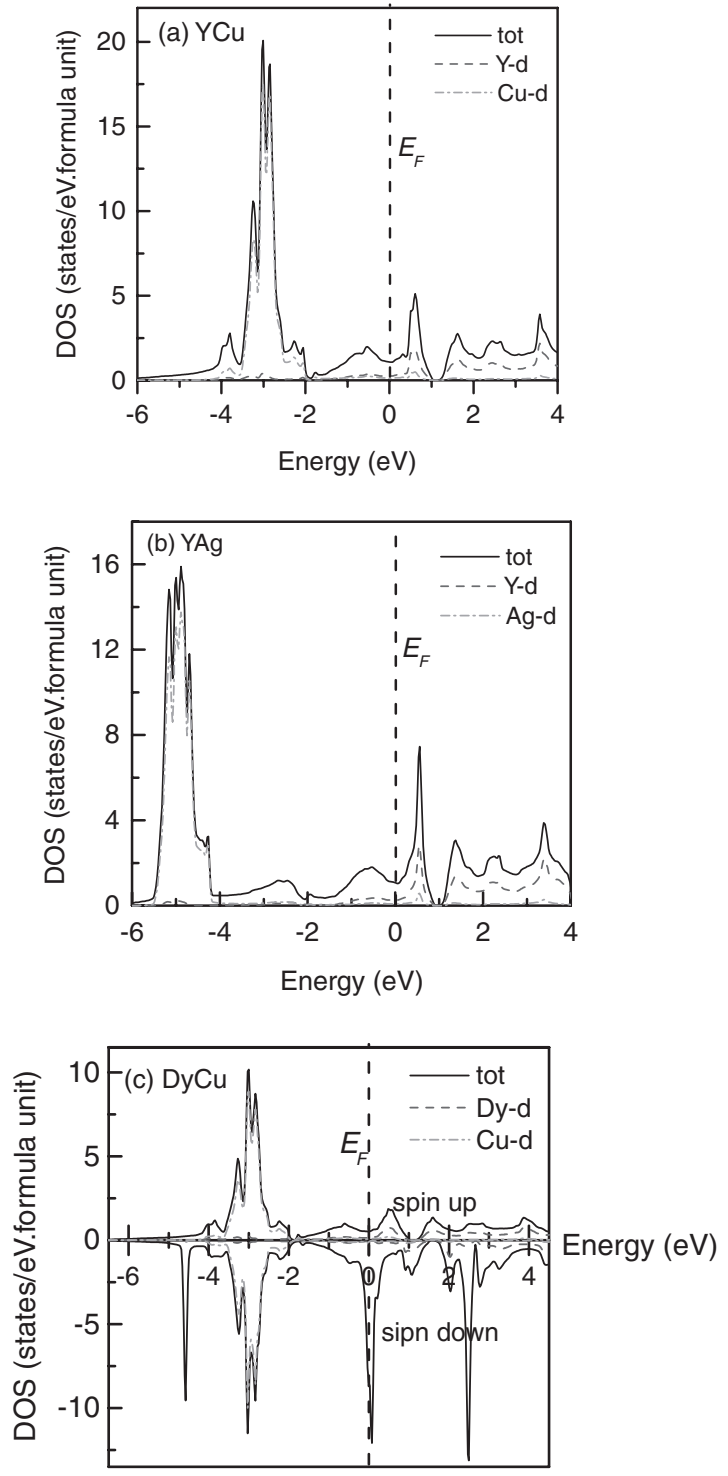

Fig. 3 The total and partial density of states of $R M$ intermetallics: (a) YCu, (b) YAg and (c) DyCu.

The polycrystalline shear modulus is associated with the resistance to plastic deformation while the bulk modulus represents the opposition to bond rupture, so the ratio $K / G$ (bulk modulus $K$ divided by the shear modulus $G$ ) may be considered as a criterion of the ductility/brittleness performance of materials. ${ }^{19)}$ This criterion is named as Pugh's criterion. For materials with melting temperature above $900^{\circ} \mathrm{C}$, its ductility (ability to plastic deformation without fracture) can be characterized by high $K / G$ ratio ( $>1.75$ ), while low $K / G$ is representative of brittleness. ${ }^{8,20)}$ The melting temperatures of $\mathrm{YCu}, \mathrm{YAg}$ and DyCu are 935, 1160 and $955^{\circ} \mathrm{C}$, respectively. They are all greater than $900^{\circ} \mathrm{C}$. Using the relation between the bulk modulus and shear modulus and the relation between Poisson's ratio $v$, and the elastic constants, the $K / G$ ratio can be calculated as follows:

$$
\begin{aligned}
\frac{K}{G} & =\frac{2}{3}\left(\frac{1+v}{1-2 v}\right) \\
v & =\frac{C_{12}}{C_{11}+C_{12}}
\end{aligned}
$$

The calculated values are 2.30 for $\mathrm{YCu}, 3.00$ for $\mathrm{YAg}$ and 2.05 for $\mathrm{DyCu}$, respectively. According to the Pugh's criterion, they should be ductility at room temperature, which is consistent with the experimental work. ${ }^{4-7)}$ The elastic anisotropy is also calculated in this work. The expression for the anisotropy is:

$$
A=2 \frac{C_{44}}{C_{11}-C_{12}}
$$

The value of the anisotropy is also listed in Table 2. Unlike other B2 intermetallics, the elastic anisotropy of $R M$ intermetallics is close to 1 , and lies between the brittle $\mathrm{B} 2$ intermetallics $(A>2)$ and $\mathrm{B} 2$ ionic compounds $(A<0.7)$, indicating a more isotropic behavior. ${ }^{8)}$ The values of the anisotropy and Poisson's ratio of the ductile $R M$ intermetallics are very close to the BCC metals (W, $A=1.00, v=0.28$; Mo, $A=0.90, v=0.29) .{ }^{4,21}$ ) The more isotropic nature of the $R M$ compounds may account for the absence of the brittleness as compared with the strong anisotropy $(A>2$ or $A<0.7)$, and provides additional support to that $R M$ intermetallics are a new class of materials, which are different from the other B2 structural intermetallics.

\section{Summary}

In conclusion, the electronic structure and elastic properties of $\mathrm{YCu}, \mathrm{DyCu}$ and $\mathrm{YAg}$ were studied by the first-principles calculations. Based on the results of density of states and elastic properties, their ductility is predicted. These ductile RM intermetallics are different from the other ordinary brittle B2 intermetallics. They exhibit more isotropic behavior, very close to the BCC elemental metals. 


\section{Acknowledgements}

This work was supported financially by the Natural Science Foundation of Jiangsu Province (Grant No. BK2007213), the PhD Programs Foundation of Ministry of Education of China (Grant No. 20040288018), and the "Qinglan Project" Foundation of Jiangsu Province. The authors also thank Ms. J. N. Cai and X. H. Yuan for proof reading.

\section{REFERENCES}

1) C. T. Liu, E. P. George, P. J. Maziasz and J. H. Schneibel: Mater. Sci. Eng. A 258 (1998) 84-98.

2) N. S. Stoloff, C. T. Liu and S. C. Deevis: Intermetallics 8 (2000) 1313 1320 .

3) D. B. Miracle: Acta Metall. Mater. 41 (1993) 649-684.

4) K. Gschneidner, A. Russell, A. Pecharsky, J. Morris, Z. Zhang, T. Lograsso, D. Hsu, C. H. Chester, Y. Y. Ye, A. Slager and D. Kesse: Nature Mater. 2 (2003) 587-590.

5) Z. Zhang, A. M. Russell, S. B. Biner, K. Gschneidner and C. C. H. Lo: Intermetallics 13 (2005) 559-564.

6) A. M. Russell, Z. Zhang, T. A. Lograsso, C. C. H. Lo, A. O. Pecharsky, J. R. Morris, Y. Ye, K. A. Gschneidner and A. J. Slager: Acta Mater. 52 (2004) 4033-4040.
7) A. M. Russell, Z. Zhang, K. A. Gschneidner, T. A. Lograsso, A. O. Pecharsky, A. J. Slager and D. C. Kesse: Intermetallics 13 (2005) 565-571.

8) J. R. Morris, Y. Y. Ye, Y. B. Lee, B. N. Harmon, K. A. Gschneidner and A. M. Russell: Acta Mater. 52 (2004) 4849-4857.

9) Y. J. Shi, Y. L. Du, G. Chen and G. L. Chen: Phys. Lett. A 368 (2007) 495-498.

10) V. I. Anisimov, F. Aryasetiawan and A. I. Lichtenstein: J. Phys.: Condens. Matter. 9 (1997) 767-808.

11) H. Harima: J. Magn. Magn. Mater. 226-230 (2001) 83-84.

12) P. Blaha, K. Schwarz, G. K. H. Madsen, D. Kvasnicka and J. Luitz: WIEN2k, An Augmented Plane Wave + Local Orbitals Program for Calculating Crystal Properties, Karlheinz Schwarz, Techn. (Univeresität Wien, Austria, ISBN 3-9501031-1-2, 2001).

13) M. Diviš and J. Kuriplach: Physica B 205 (1995) 353-364.

14) K. B. Panda and K. S. Ravi Chandran: Acta Mater. 54 (2006) 16411657.

15) B. B. Karki, L. Stixrude, S. J. Clark, M. C. Warren, G. J. Ackland and J. Crain: Am. Mineralogist 82 (1997) 51-60.

16) F. D. Murnaghan: Proc. Natl. Acad. Sci. USA 30 (1944) 244-247.

17) J. Y. Wang and Y. C. Zhou: Phys. Rev. B 69 (2004) 144108-144102.

18) Y. Wu and W. Hu: Eur. Phys. J. B 60 (2007) 75-81.

19) S. F. Pugh: Philos. Mag. 45 (1954) 823-844.

20) L. Vitos, P. A. Korzhavyi and B. Johansson: Phys. Rev. Lett. 88 (2002) 155501-155504.

21) Y. G. Zhang, Y. F. Han, G. L. Chen, J. T. Guo, X. J. Wang and D. Feng: Structural materials of intermetallic compounds. Beijing, China, ISBN 7-118-02321-3, 2001. 